\title{
Magnetic relaxation studies on a single-molecule magnet by time-resolved inelastic neutron scattering
}

\author{
O. Waldmann, G. Carver, C. Dobe, D. Biner, A. Sieber, and H. U. Güdel \\ Department of Chemistry and Biochemistry, University of Bern, CH-3012 Bern, Switzerland \\ H. Mutka and J. Ollivier \\ Institut Laue-Langevin, 6 rue Jules Horowitz, BP 156, 38042 Grenoble Cedex 9, France \\ N. E. Chakov \\ Department of Chemistry, University of Florida, Gainesville, Florida 32611, USA
}

(Dated: November 6, 2018)

\begin{abstract}
Time-resolved inelastic neutron scattering measurements on an array of single-crystals of the single-molecule magnet $\mathrm{Mn}_{12}$ ac are presented. The data facilitate a spectroscopic investigation of the slow relaxation of the magnetization in this compound in the time domain.

PACS numbers: 33.15.Kr, 71.70.-d, 75.10.Jm
\end{abstract}

A detailed understanding of the magnetic relaxation in small magnetic particles is of fundamental importance for their application. The discovery of magnetic bistability at the molecular level in polymetallic clusters such as $\mathrm{Mn}_{12}$ ac, the so-called single-molecule magnets (SMMs), has opened new possibilities $\frac{1}{1}$ The slow relaxation of the magnetization in SMMs below a blocking temperature $T_{B}$ is associated with a large ground-state spin $S$ and easy-axis anisotropy $D M^{2}$ ( $S$ and $M$ are the total spin and magnetic quantum numbers, and $D<0$ ). The $2 S+1$ levels of the spin ground state are thus split, as indicated in Fig. 2a, producing an energy barrier of height $\Delta \approx$ $-D S^{2}$ separating the up and down orientations of the $\operatorname{spin}\left(\right.$ for $\mathrm{Mn}_{12}$ ac, $S=10, \Delta=67 \mathrm{~K}, T_{B} \approx 3.5 \mathrm{~K}$ ) ! $\frac{1}{?}$

The relaxation behavior of SMMs has been studied by various techniques, such as magnetization measurements, nuclear magnetic resonance, and frequency-domain electron spin resonance ${ }^{1.2}$ In this work are presented timeresolved inelastic neutron scattering (INS) measurements on an array of single-crystals of $\mathrm{Mn}_{12}$ ac. Time-resolved INS has been applied previously to the study of rotational tunneling phenomena ${ }^{3}$. It is shown here that this approach, due to recent improvements in INS spectrometry, can be applied to the study of magnetic relaxation.

Needle-shaped single crystals of non-deuterated $\left[\mathrm{Mn}_{12} \mathrm{O}_{12}\left(\mathrm{CH}_{3} \mathrm{COO}\right)_{16}\left(\mathrm{H}_{2} \mathrm{O}\right)_{4}\right], \mathrm{Mn}_{12}$ ac, with approximate dimensions $5 \times 0.5 \times 0.5 \mathrm{~mm}^{3}$ and mass ca. $2 \mathrm{mg}$ were obtained following literature procedures $\underline{4}$ $\mathrm{Mn}_{12}$ ac crystallizes in the space group $\mathrm{I} \overline{4}$; the magnetic anisotropy axis $z$ is coincident with the needle axis. This permitted the construction of a sample consisting of an array of many (ca. 500) single crystals, aligned in grooves (dimensions: $13 \times 0.6 \times 0.6 \mathrm{~mm}^{3}, 16$ grooves per platelet) milled into $1 \mathrm{~mm}$ thick $15 \times 15 \mathrm{~mm}^{2}$ aluminium platelets (Fig. 1a). A total of 15 platelets were stacked in an aluminium container (Fig. 1b), and sealed under $\mathrm{He}$ with an indium seal and aluminium screws.

Inelastic neutron scattering (INS) measurements were performed on the time-of-flight spectrometer IN5 at the
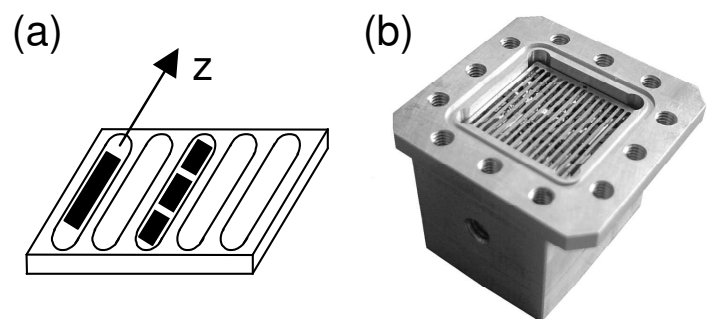

FIG. 1: (a) Schematic of an aluminium platelet. The black bars depict $\mathrm{Mn}_{12}$ ac crystals positioned in the grooves, such that the anisotropy axes $z$ (= needle axes) are oriented along the grooves. (b) Photograph of the aluminium box containing the platelets filled with $\mathrm{Mn}_{12}$ ac crystals.

Institut Laue-Langevin in Grenoble, France. The sample was inserted into an orange ${ }^{4} \mathrm{He}$ cryostat equipped with a $2.5 \mathrm{~T}$ vertical-field magnet. The sample was aligned with the $z$ axes of the crystals parallel to the magnetic field. The field was changed at the maximum ramp rate provided by the power supply $(4 \mathrm{mT} / \mathrm{s}$ for fields above ca. $0.2 \mathrm{~T}$, but significantly slower for lower fields). An initial neutron wavelength of $5.9 \AA$ was used, affording a resolution of $60 \mu \mathrm{eV}$ at the elastic line. In this energy range, the background of aluminum is negligible. The data were corrected for detector efficiency using a vanadium standard. The spectra shown below correspond to the sum over all the scattering angles.

In the following the dependence of the $\mathrm{Mn}_{12}$ ac INS spectrum on a magnetic field $B$ applied parallel to the $z$ axis shall first be discussed. Measurements were made by first cooling the sample in zero field from $5 \mathrm{~K}$, i.e., above the blocking temperature, to $1.5 \mathrm{~K}$. Accordingly, the lowest levels on both sides of the double-well potential, i.e., the $M= \pm 10$ levels, were equally populated, see Fig. 2a (the population densities of the $M= \pm 10$ levels are denoted as $\left.n_{ \pm}\right)$. The field was then increased stepwise. The resulting spectra are shown in Fig. 2d. The zero-field spectrum shows the known peak at $E_{0}=1.24 \mathrm{meV}$, corre- 
(c)

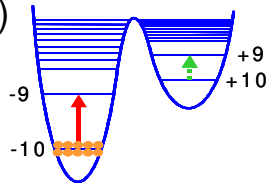

(b)

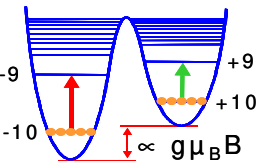

(a)

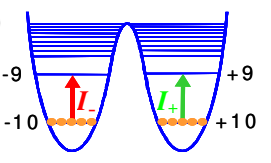

(d)

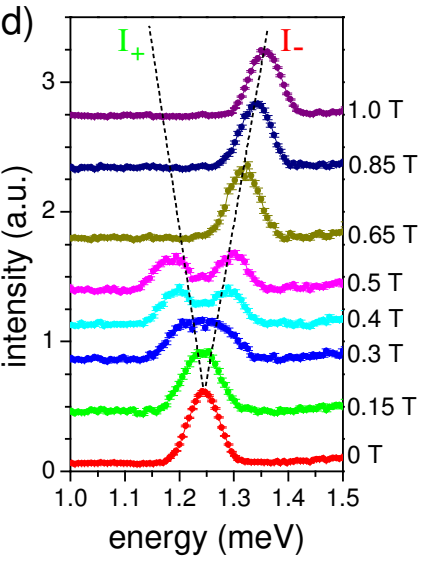

FIG. 2: (a-c) Schematics showing the transitions observed for (a) $B=0$, (b) $0<B \leq 0.5 \mathrm{~T}$, and (c) $B>0.5 \mathrm{~T}$. Transitions $I_{-}$and $I_{+}$are indicated by arrows. Circles symbolize the populations of the $M=-10$ and $M=+10$ levels. (d) $1.5 \mathrm{~K}$ neutron-energy-loss spectra for the magnetic fields indicated. Curves are offset for clarity.

sponding to the two transitions $M=-10 \rightarrow-9\left(I_{-}\right)$and

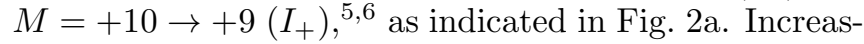
ing the field leads to a tilting of the double-well potential due to the Zeeman energy, see Fig. 2b. The energies of the two transitions $I_{-}$and $I_{+}$are now different, they appear at $E_{-}=E_{0}+g \mu_{B} B$ and $E_{+}=E_{0}-g \mu_{B} B$, respectively $\left(g=2.0, \mu_{B}\right.$ is the Bohr magneton). The experimental data for $B \leq 0.5 \mathrm{~T}$ indeed exhibit two peaks at exactly $E_{-}$and $E_{+}$, see Fig. 2 d. Note that for the fields used here the level $M=+10$ lies considerably above the ground state, e.g., $20 g \mu_{B} B=0.93 \mathrm{meV}$ at $0.4 \mathrm{~T}$, and would thus normally be depopulated. The observation of both transitions $I_{-}$and $I_{+}$thus directly reflects the slow relaxation of the magnetization in $\mathrm{Mn}_{12}$ ac at $1.5 \mathrm{~K}$, which permits the metastable state with the $M=+10$ level populated. For $B>0.5 \mathrm{~T}$, however, the system completely relaxed back to the ground state due to the crossing of the $M=+10$ and $M=-9$ levels (Fig. 2c). Accordingly, only the $I_{-}$peak was observed in this field range. Interestingly, the linewidths are resolution limited, indicating an alignment of the crystals within $5^{\circ}$. This is possibly because of a self alignment of the crystals due to the torque exerted by an applied magnetic field.

The above experiments clearly show i) that for $B \leq$ $0.5 \mathrm{~T}$ it is possible to create a metastable situation of the populations and ii) that for $B>0$ the populations of the $M=+10$ and $M=-10$ levels can be measured individually via the intensities of the transitions $I_{-}$and $I_{+}$ appearing at different transfer energies. This permitted a time-resolved study of the magnetic relaxation in $\mathrm{Mn}_{12}$ ac as follows: First, only the $M=+10$ level was populated by applying a negative field of $-0.85 \mathrm{~T}$. Then, the field was swept quickly to a positive value $B>0 \leq 0.5 T$, so that all molecules were in the now higher lying $M=+10$ level. This triggered the relaxation of the system towards

(a)

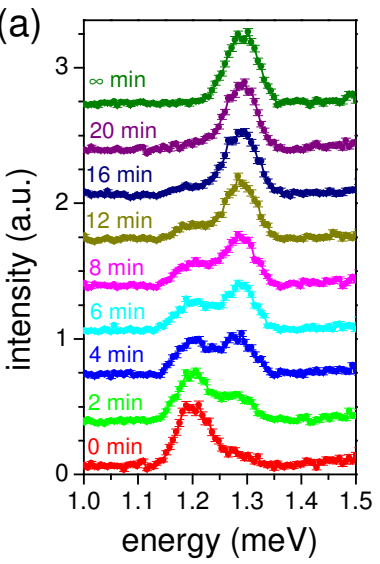

(b)

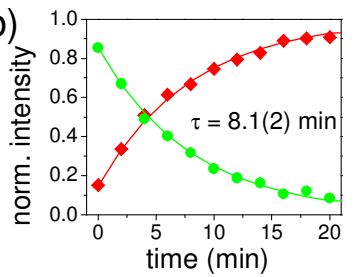

(c)

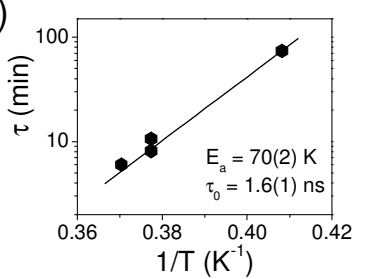

FIG. 3: (a) Neutron energy-loss spectra at $2.65 \mathrm{~K}$ as measured with the s\&g protocol (see text). Curves are offset for clarity. (b) Time dependence of the normalized intensities of the transitions $I_{-}$(squares) and $I_{+}$(circles). Solid curves correspond to a fit to an exponential decay with a relaxation time $\tau$ as indicated. (c) Arrhenius plot for the relaxation times. Circles are experimental points, the line is a fit to the Arrhenius law $\tau=\tau_{0} e^{E_{a} /\left(k_{B} T\right)}$ with parameters as indicated.

the $M=-10$ level, which was followed by repeated INS measurements (a run time of $\Delta t=5$ min already gave reasonable spectra; typically, however, $\Delta t=30 \mathrm{~min}$ was chosen). In $\mathrm{Mn}_{12}$ ac the relaxation time is some years at $1.5 \mathrm{~K}$, and a few seconds at the blocking temperature. Thus, through varying the temperature, the relaxation time could be tuned such that time slices of $\Delta t=5$ $30 \mathrm{~min}$ allowed reasonable time resolution. Specifically, time-resolved INS spectra were recorded for $T=2.45$, 2.65 , and $2.70 \mathrm{~K}$ with $B=0.4 \mathrm{~T}$ (data not shown). At $B=0.4 \mathrm{~T}$, the $I_{-}$and $I_{+}$transitions appear at transfer energies of $E_{-}=1.29 \mathrm{meV}$ and $E_{+}=1.19 \mathrm{meV}$, respectively, see Fig. 2d.

A more refined measurement protocol, denoted stopand-go (s\&g), allows for an improvement over the above constant- $T$ protocol. Here, the temperature is set to a value $T^{*}$ much lower than the temperature $T$ at which the relaxation is to be studied, such as to essentially freeze the system. After initialization steps as above (but at $\left.T^{*}\right)$, the relaxation is followed by repeatedly setting the temperature to the target temperature $T$, waiting for a time $\delta t$, cooling back to $T^{*}$, and measuring for a time $\Delta t$. Since the relaxation time at $T^{*}$ is much longer than at $T$, this amounts to taking snapshots of the population distribution at intervals of $\delta t$. The possibility of freezing a state during recovery to equilibrium thus permits both short time intervals and reasonable acquisition times. The time-resolved INS spectrum was recorded with the $\mathrm{s} \& \mathrm{~g}$ protocol at $T=2.65 \mathrm{~K}$ with $\delta t=2 \mathrm{~min}$, see Fig. 3a $\left(B=0.4 \mathrm{~T}, T^{*}=2.3 \mathrm{~K}, \Delta t=30 \mathrm{~min}\right.$; an animated movie is provided in the supporting material).

The experimental data clearly allow one to follow the relaxation of the populations of the $M= \pm 10$ levels. The time dependence of the peak intensities were ana- 


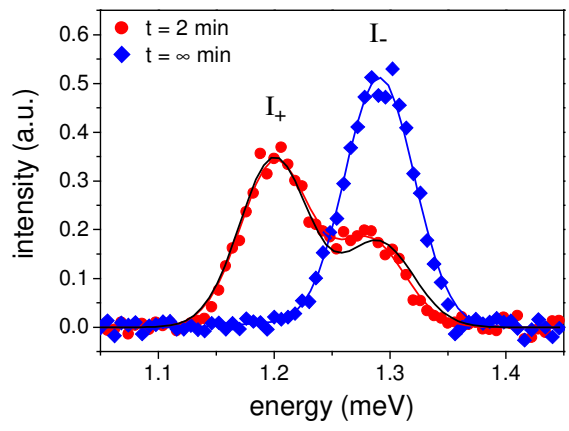

FIG. 4: INS spectra after $2 \mathrm{~min}$ and long waiting times recorded at $2.65 \mathrm{~K}$ using the $\mathrm{s} \& \mathrm{~g}$ protocol. Solid lines are best-fit curves, for details see the text.

lyzed quantitatively by fitting the data at each time step to two Gaussians with a constant offset. In the fits, the linewidths of the Gaussians were fixed to the spectrometer resolution. The values obtained for the peak intensities were then normalized such as to add up to 1 on average. The result is shown in Fig. 3b. The time dependence of the peak intensities, or populations $n_{-}$and $n_{+}$, respectively, are well described by an exponential decay, $n_{-}(t)=\exp (-t / \tau)$ and $n_{+}(t)=1-n_{-}(t)$, as demonstrated by the solid lines in Fig. 3b. For the current experiments, the population was not inverted completely after the initialization step, i.e., about $15 \%$ of the molecules remained in the ground state (this is evident from the 0 min curve in Fig. 3a, which shows small intensity also at $1.29 \mathrm{meV}$ ). This is attributed to the slow ramp rates provided by the magnet power supply near zero field.

The relaxation times obtained from the exponential decay fits were $8.1(2) \mathrm{min}$ for the $\mathrm{s} \& \mathrm{~g}$ experiment at $2.65 \mathrm{~K}$, and $74(2), 10.6(7)$, and 6.0(5) min for the constant- $T$ experiments at $2.45,2.65$, and $2.70 \mathrm{~K}$, respectively. The relaxation times obtained by the $\mathrm{s} \& \mathrm{~g}$ and constant- $T$ protocols at $2.65 \mathrm{~K}$ are in good agreement. Plotting the relaxation time as a function of the inverse temperature (Arrhenius plot, Fig. 3c), reveals a thermally activated behavior consistent with thermally-assisted tunneling of $\mathrm{Mn}_{12}$ ac in this temperature range $\frac{1}{\underline{1}}$ The activation energy $E_{a}=70(2) \mathrm{K}$, thus determined, is slightly larger than the zero-field value of $62 \mathrm{~K}, \frac{1}{1}$ Zero-field relaxation measurements on SMMs generally yield $E_{a}<\Delta$, which has been explained by efficient tunneling paths near the top of the barrier $\stackrel{\underline{1}}{\underline{n}}$ In the present case, however, the Zeeman energy removes these paths increasing $E_{a}$.

The data, allowing also for a time-resolved investigation of the peak positions, revealed an interesting effect. After initialization, both peaks were shifted slightly towards the center $E_{0}$ as compared to their equilibrium positions $E_{ \pm}=E_{0} \pm g \mu_{B} B$. The shifts were small, about $8 \mu \mathrm{eV}$, but statistically significant. This is illustrated by the s\&g-INS spectra of Fig. 3a at $2 \mathrm{~min}$ and after long waiting times, presented in more detail in Fig. 4. The two solid lines which fit the data well correspond to best-fit curves, where both intensities and positions were fitted. The third curve, which approximates the 2 min data with less accuracy, was obtained from a fit but with the transfer energies fixed to the equilibrium values. The shift of the $I_{-}$peak at ca. $1.29 \mathrm{meV}$ towards lower energies is clearly visible. The observations may be described in terms of a time-dependent effective magnetic field $B(t)=B_{e q}-\Delta B(t)$, which differs from the field at equilibrium $B_{e q}$. The experiments yield $\Delta B(0) \approx 70 \mathrm{mT}$. As a function of time, the peak positions approached their equilibrium values, but the limited statistics of the data did not allow a precise determination of the relaxation law. The effect described has been observed in all measurements with similar values for $\Delta B(0)$. It might seem natural to associate $\Delta B(t)$ with the magnetization $M=B-H$ (in units of T). However, for needles the depolarization factor $N=1$ and the essentially cubic arrangement of the $\mathrm{Mn}_{12}$ ac molecules in the crystals means that the local magnetic field is equal to the external magnetic field. Further studies are necessary to elucidate the details of this phenomenon.

In conclusion, we have demonstrated that with highperformance time-of-flight spectrometers, such as IN5 at the ILL, the magnetic relaxation processes in magnetic compounds can be studied with time-resolved INS measurements. We believe that the spectroscopic resolution provided by this methodology will allow unprecedented insights into the physics of magnetic relaxation.

\section{Acknowledgments}

Financial support by EC-RTN-QUEMOLNA, contract $\mathrm{n}^{\circ}$ MRTN-CT-2003-504880, and the Swiss National Science Foundation is acknowledged.
${ }^{1}$ D. Gatteschi and R. Sessoli, Angew. Chem. Int. Ed. 42, 268 (2003).

2 M. Dressel, B. Gorshunov, K. Rajagopal, S. Vongtragool, and A.A. Mukhin, Phys. Rev. B 67, 060405 (2003).

${ }^{3}$ R. Mukhopadhyay, J. Tomkinson, and C.J. Carlile, Physica B 226, 171 (1996).

${ }^{4}$ T. Lis, Acta Crystallogr. Sect. B 362042 (1980).
${ }^{5}$ I. Mirebeau, M. Hennion, H. Casalta, H. Andres, H. U. Güdel, A. V. Irodova, and A. Caneschi, Phys. Rev. Lett. 83, 628 (1999).

${ }^{6}$ R. Bircher, G. Chaboussant, A. Sieber, H. U. Güdel, and H. Mutka, Phys. Rev. B 70, 212413 (2004). 